Juhan Kreem (Tallinn)

\title{
NETZWERKE UM JASPER VON MUNSTER. DER DEUTSCHE ORDEN WÄHREND DER LIVLÄNDISCHEN KOADJUTORFEHDE IM JAHRE 1556
}

KEYWORDS

political history, administration, conflict, Teutonic Order, Livonia, WarofCoadjutor's

目 ie ${ }^{1}$ Koadjutorfehde ist bekanntlich der Anfang vom Ende des mittelalterlichen Livlands. Am Vorabend des Livländischen Krieges kam es zu inneren Streitigkeiten um die Koadjutorwahl des Rigaer Erzbischofs. Obwohl sich die livländischen Stände im Jahre 1546 in Wolmar ein Mitbestimmungsrecht bei der Wahl eines fürstlichen Koadjutors gesichert hatten, beharrte Erzbischof Wilhelm auf der Freiheit des Stiftes und wollte Christoph von Mecklenburg zu seinem Koadjutor wählen lassen. Die allgemeine Empörung in Livland ermöglichte es dem Deutschen Orden im Sommer 1556, einen erfolgreichen Kriegszug gegen den Erzbischof zu führen, Wilhelm zusammen mit seinem Koadjutor abzusetzen und gefangen zu nehmen. Nur die Intervention des Königs von Polen, Sigismund August, drängte den Orden zum Rückzug. Der Erzbischof wurde restituiert und der Orden war gezwungen, im September 1557 in Poswol einen Frieden mit dem König zu schließen. ${ }^{2}$

1 Verwendete Abkürzungen: SRA = Svensk Riksarkivet (Schwedisches Reichsarchiv); TLA = Tallinna Linnaarhiiv (Stadtarchiv Tallinn). Dieser Aufsatz wurde von der Estnischen Wissenschaftsagentur durch den Grant Nr. IUT 18-2 unterstützt.

2 Eine umfassende neue Spezialuntersuchung zum Thema fehlt. Die neueren Beiträge behandeln die Koadjutorfede in einem größeren Zusammenhang oder nur einen Teilaspekt davon: T. Lange, Zwischen Reformation und Untergang Alt-Livlands. Der Rigaer Erzbischof 
Gleichzeitig mit dem Kampf um den Koadjutor des Erzbischofs entbrannte aber innerhalb des Deutschen Ordens in Livland auch eine eigene „Koadjutorfehde“. Für den altersschwachen Ordensmeister Heinrich von Galen wurde der Komtur zu Fellin, Wilhelm von Fürstenberg, zum Koadjutor gewählt, wogegen der Landmarschall des Ordens in Livland, Jasper von Munster, der selber das Amt bekleiden wollte, im Frühling 1556 protest erhob. Der Landmarschall wurde wegen seines Ungehorsams abgesetzt und musste nach Kaunas in Litauen fliehen. Von dort her kämpfte er (mit Hilfe von Herzog Albrecht von Preußen und König Sigismund August von Polen) vergeblich um seine Restitution. Nach vielen Jahren musste sich Jasper mit der vom polnischen König vergebenen bescheidenen Grenzfestung Ascheraden als Vergütung begnügen.

Der ganze Vorfall um Jasper von Munster ist kürzlich von Hans Mol aus der Perspektive des Landmarschalls gründlich dargestellt worden. ${ }^{3}$ Mol stellt die familiären Verbindungen von Jasper dar und analysiert die Beweggründe seines Handelns. Unter den Motiven Jaspers setzt Mol die Sorge um Livlands Zukunft an die erste Stelle. Der Hauptstreitpunkt war nach Mol die Bündnispolitik Livlands, wobei Jasper eine Annäherung an Polen-Litauen und Schweden befürwortete, um der „Russischen Gefahr“ zu widerstehen, seine Kontrahenten aber mit Wilhelm von Fürstenberg an der Spitze betrieben wegen ihrer Feindschaft zu Polen-Litauen Friedenspolitik mit den Moskoviten. Ganz zu Recht hebt Mol hervor, dass bei Jaspers Scheitern seine Weigerung, Wilhelm von Fürstenberg als Koadjutor anzuerkennen, sowie seine Verbindungen zu Erzbischof Wilhelm eine entscheidende Rolle spielten. ${ }^{4}$

Dieser Beitrag macht sich zur Aufgabe, den Fokus von der großen Politik auf die inneren Strukturen des Ordens in Livland zu verschieben. Meine Frage gilt

Wilhelm von Brandenburg im Beziehungsgeflecht der livländischen Konfoderation und ihrer Nachbarländer, Hamburg 2014, S. 188-242; U. Müller, Erzbischof Wilhelm von Riga und die Reformation in Livland 1535-1563, in: Preussen und Livland im Zeichen der Reformation, hrsg. v. A. Mentzel-Reuters, K. Neitmann, Osnabrück 2014, S. 241-343; M. Maasing, Saare-Lääne ja koadjuutorivaenus: keskaegse Liivimaa viimased kodusöjad [The feud of Ösel-Wiek and the Coadjutor's feud: the last civil wars in medieval Livonia], Ajalooline ajakiri 2010, 2, S. 115-151; V. E. Popov, A. I. Filjuškin, »Voina koad'jutorov《 i Pozvol'skie soglašenija 1557 g. [Koadjutorfehde und die Verträge von Poswol 1557], Studia Slavica et Balcanica 2009, 1/2, S. 151-184; S. Hartmann, Neue Quellen zur livländischen Koadjutorfehde 1555/56, in: Aus der Geschichte Alt-Livlands. Festschrift für Heinz von zur Müblen zum 90. Geburtstag, hrsg. v. B. Jähnig, K. Militzer, Münster 2004, S. 275-306.

3 H. J. Mol, Verrader van Lijfland? De Nederlandse ordemaarschalk Jasper van Munster en het begin van de Lijflandse crisis, 1554-1557 [Traitor to Livonia? The Duch Land Marshal of the Order Jasper van Munster and the outset of the Livonian crisis, 1554-1556], in: ders., Vechten, bidden en verplegen, Hilversum 2011, S. 235-268 (vgl. in diesem Band, S. 113-148])

4 Ebd., S. 267. 
den unterschiedlichen politischen und administrativen Netzwerken innerhalb des Ordens. Konkret wäre es wichtig die Natur des Netzwerks um Jasper von Munster zu bestimmen: Welche Beziehungen hatte er und wie wäre seine unmittelbare Anhängerschaft zu charakterisieren? Zweitens muss gefragt werden, wie die Entmachtung Munsters möglich war. Welche Strukturen und Kräfte waren es, die entscheidend für Jaspers Scheitern waren? Uns interessiert hier also kurzgesagt das innere Funktionieren des Deutschen Ordens im 16. Jahrhundert.

Ein Netzwerk soll in dieser Analyse im weiteren Sinne als eine Struktur aus Knotenpunkten und Verbindungen verstanden werden. Mit Knotenpunkten sind hier agierende Menschen gemeint, die durch unterschiedliche, formelle und informelle, Beziehungen in eine Vielfalt von Netzwerken eingebunden sind bzw. diese auch aktiv mitgestalten. Obwohl die Worte Struktur und Netzwerk statische Gebilde suggerieren, handelt es sich bei einem Netzwerk um einen dynamischen Prozess, wobei die Stärke der geschaffenen Verbindungen immer wieder von weiteren Kräften auf die Probe gestellt wird. Die Netzwerke entstehen, wandeln oder lösen sich auf.

Einige Worte zur Quellenlage. Die Rigische Koadjutorfehde wurde schon anhand der Mecklenburgischen Überlieferung in Schwerin von Alexander Bergengrün gründlich dargestellt. ${ }^{5}$ Die wichtigste Ergänzung dazu ist das preußische Herzogliche Briefarchiv, heute in Berlin, in dem neben unterschiedlichen Verhandlungsunterlagen der umfangreiche Briefwechsel Jaspers, seiner Anhänger, Erzbischof Wilhelms und Herzog Albrechts erhalten ist. Diese Kommunikation ist durch die Regestenpublikation von Stefan Hartmann jetzt besser zugänglich gemacht worden ${ }^{6}$ und wurde auch schon von Mol in Bezug auf Jasper ausgewertet. Die Unterlagen des Königsberger Archivs, obwohl zum Teil auch sehr detailliert, vermitteln vor allem den Standpunkt Munsters. Darüber hinaus ist zu bemerken, dass der größere Teil der Materialien über Munster nach den eigentlichen Ereignissen entstanden ist, sich also mit den Erklärungen ex post beschäftigt. Demgegenüber sind die Belege über die Aktionen und Argumente der Kontrahenten Munsters äußerst spärlich. Die innere Kommunikation des Deutschen Ordens in Livland ist wegen des Verlusts der Ordensarchive überhaupt schwer zu erfassen. Einige bisher unbekannte Aktenstücke zu unseren Thema kann man jedoch unter den spärlichen Überresten des ordensmeisterlichen Archivs in der Livonica-Sammlung des Schwedischen Reichsarchivs finden.

5 A. Bergengrün, Herzog Christoph von Mecklenburg, letzter Koadjutor des Erzbistums Riga. Ein Beitrag zur livländischen und Mecklenburgischen Geschichte, Reval 1898.

6 Herzog Albrecht von Preußen und Livland, bearb. v. S. Hartmann, U. Müller, 7 Bde., Köln-Weimar-Wien 1996-2008. 


\section{JASPERs NetzWerke}

Fangen wir mit der Netzwerkbildung Jaspers von Munster an. Die Spannungen um die Leitung des Ordens hatten beim Ausbruch des Konflikts schon eine Weile bestanden. Heinrich von Galen war sehr alt, und man spekulierte öffentlich über seine Nachfolge. Am Anfang des Jahres 1555 wusste Erzbischof Wilhelm, dass neben dem Landmarschall auch der Komtur von Fellin, Wilhelm von Fürstenberg ${ }^{7}$, und der Komtur von Goldingen, Christoph von Neuhof gen. Ley ${ }^{8}$, Meister werden wollten. Damals bekam Erzbischof Wilhelm auf Umwegen das Signal, dass Jasper mit Unterstützung Wilhelms seine Wahl durchsetzten wollte.9 In einem anderen Brief aus dieser Zeit berichtet Wilhelm, dass auch der Komtur von Goldingen zu ihm übertreten wollte. ${ }^{10}$ Davon hören wir aber nichts mehr, und irgendwann während des Jahres 1555 wurden gerade die Kontakte Jaspers von Munster zu Erzbischof Wilhelm und dessen Bruder Herzog Albrecht von Preußen intensiver. Anfang 1556 fungierte Albrecht schon als Bürge für eine Geldanleihe an Jasper. ${ }^{11}$ Diese Verbindungen zu Wilhelm und Albrecht können nicht anders bewertet sein, als dass Jasper versuchte Kräfte außerhalb des Ordens für seine eigene Ziele zu mobilisieren. Ob nun auf Jaspers Agenda das Beste Livlands oder seine persönliche Karriere ganz oben stand, können wir hier in unserem Zusammenhang dahingestellt lassen; seit Anfang 1556 war er jedenfalls durch sein Bündnis mit Hohenzollernbrüder auf Konfliktkurs. ${ }^{12}$

Über die Beziehungen Jaspers außerhalb des Ordens sind wir durch Erzbischof Wilhelm relativ gut unterrichtet. Wir wissen aber kaum etwas über Jaspers Parteigänger innerhalb des Ordens. Wenn es in Bezug auf die Ordensüberlieferung verständlich ist, dass nach dem Sturz des Landmarschalls die Verbindungen zu ihm lieber verdeckt wurden, hat uns Jasper von Munster selbst auch nicht viele Personen genannt, die als seine Mitstreiter zu charakterisieren wären. Nach seiner Flucht stand Jasper im Briefkontakt mit dem Vogt von Bauske, Jobst von Walrave ${ }^{13}$, und dem Komtur zu Marienburg, Philipp Schall von Bell ${ }^{14}$, und versuchte vergeb-

7 Ritterbrüder im livländischen Zweig des Deutschen Ordens, hrsg. v. L. Fenske, K. Militzer, Köln-Weimar-Wien 1993, Ritterbrüder, Nr. 277.

8 Ebd., Nr. 616.

9 Herzog Albrecht von Preußen und Livland (wie Anm. 6), Bd. (1551-1557), Nr. 1660/1.

10 Ebd., Nr. 1680.

11 Ebd., Nr. 1761.

12 Mol (wie Anm. 3), S. 267, stellt Fürstenberg als denjenigen dar, der als erster zur Eskalation überging.

13 Ritterbrüder (wie Anm. 7), Nr. 932.

14 Ebd., Nr. 761. 
lich, sie als Vermittler zu gewinnen. ${ }^{15}$ In einem späteren Schreiben nennt Jasper Gotthard Kettler (den Komtur von Dünaburg) ${ }^{16}$ oder Gert Huin von Amstenrade (den Vogt von Wesenberg) ${ }^{17}$ als bessere Kandidaten für die Koadjutorschaft. ${ }^{18}$ Alle diese Angaben müssen aber nicht unbedingt bedeuten, dass die genannten Männer Parteigänger Munsters gewesen sind. Unter diesen war Philipp Schall von Bell im Übrigen aktiv am Kriegszug gegen Erzbischof Wilhelm beteiligt, und ihm wurde sogar die Gefangenhaltung des abgesetzten Erzbischofs anvertraut. ${ }^{19}$ Somit genoss Philipp damals genügend Vertrauen seitens der Ordensleitung. Dasselbe muss man auch für Gotthard Kettler gelten lassen. Er wurde im selben dramatischen Frühjahr 1556 mit einer wichtigen Mission nach Deutschland gesandt ${ }^{20}$, was ganz ohne Vertrauen nicht denkbar gewesen wäre. Kettler hat später bei seinem Aufstieg ins Meisteramt skrupellos gegen Wilhelm von Fürstenberg Politik gemacht, im Jahre 1556 hat er aber zur damaligen Ordensleitung gehalten und sich nicht Jasper von Munster angeschlossen, und er hat auch später nichts für ihn getan. Ebenfalls ist kein anderer Ordensgebietiger für Munster eingetreten.

Das höchste Ordensmitglied, das sich aktiv Jasper von Munster anschloss und mit ihm auch fliehen musste, war Johann von Hoete. ${ }^{21}$ Er war bis 1554 Hauskomtur zu Marienburg und hat unterschiedliche diplomatische Aufträge für den Orden ausgeführt. Nach seinen eigenen Worten gehörte er seit 1531 dem Orden an, aber die Stelle auf der Marienburg genügte seinen Ansprüchen nicht. Er verließ die Marienburg und wurde von Jasper von Munster gegen einen Jahreslohn mit 6 Pferden in den Dienst genommen. ${ }^{22}$ Er ist also kein Gebietiger gewesen und ist während der Koadjutorfehde als Diener im Hof Jaspers zu bezeichnen, ein Status, der auf einen Wandel des geistlichen Ritterordens in eine frühneuzeitliche Adelskorporation hindeutet.

15 Herzog Albrecht von Preußen und Livland (wie Anm. 6), Bd. (1551-1557), Nr. 1910/5 (Jasper von Munster an Vogt von Bauska 7. Mai 1556, Kokenhusen); ebd., Nr. 1907 (undatierte Schreiben an Komtur von Marienburg Schall von Bell, nach Nr. 1910/3 kann das mit 8. Mai Kokenhusen datiert werden), siehe auch Caspar von Münster's Vertheidigungsschrift, in: Mittheilungen aus dem Gebiete der Geschichte Liv-, Ehst- und Kurlands 10 (1861), S. 111-160, hier S. 153; Herzog Albrecht von Preußen und Livland (wie Anm. 6), Bd. (1557-1560), Nr. 2135.

16 Ritterbrüder (wie Anm. 7), Nr. 489.

17 Ebd., Nr. 454.

18 Herzog Albrecht von Preußen und Livland (wie Anm. 6), Bd. (1551-1557), Nr. 1910/1.

19 Ebd., Nr. 1939, 1986, 1979.

20 Kettlers Abreise ist datiert mit 27. Januar, vgl. Herzog Albrecht von Preußen und Livland (wie Anm. 6), Bd. (1551-1557), Nr. 1780.

21 Ritterbrüder (wie Anm. 7), Nr. 415.

22 Herzog Albrecht von Preußen und Livland (wie Anm. 6), Bd. (1557-1560), Nr. 2137. 
Jasper von Munster nennt noch flüchtig die weltlichen Ordensräte Philipp von der Brüggen und Johann Bockhorst als seine Gesinnungsgenossen für die Annäherung an Polen ${ }^{23}$, über deren Verhalten in der Koadjutorfehde ist aber nichts bekannt. In den polemischen Schriften dieser Zeit kommen noch weitere Namen vor wie zum Beispiel der ehemalige Ordenskanzler Christopher Bötticher ${ }^{24}$, er aber war mit dem Orden wegen seiner Entlohnung schon früher in Konflikt geraten und ins Exil gegangen. ${ }^{25}$ Er schloss sich demnach dem Landmarschall erst nach dessen Absetzung und Flucht an. Wenn wir jetzt zusammenfassend diese Reihe mustern, ist es schwer innerhalb der Ordenskreise eine Partei von Jasper von Munster zu sehen. Unter den Mitgliedern des Ordens mag er für die Außenpolitik Livlands Gleichgesinnte gehabt haben, sein Netzwerk unter den Gebietigern und Ordensbeamten war aber nicht besonders umfangreich und beeindruckend.

\section{Jaspers BRUCh Mit Dem ORden}

Die Stärke dieses Netzwerkes wird für uns aus den eigentlichen Aktionen deutlich. Der entscheidende Streitpunkt zwischen Jasper und dem Orden war die Koadjutorwahl für den Orden Anfang 1556. Jasper datiert dies auf den Landtag im März ${ }^{26}$; es ist aber auch möglich, dass Wilhelm Fürstenberg schon am Anfang des Jahres zum Ordenskoadjutor gewählt wurde. ${ }^{27}$ Als Gremium für eine Koadjutorwahl kommt jedenfalls nicht der Landtag, sondern nur das Ordenskapitel in Frage, auch wenn die Gebietiger ihr Kapitel während des Landtages abgehalten haben könnten. Wie dem auch sei, Fürstenberg ist nach dem Landtag im März als Koadjutor allgemein bekannt geworden. ${ }^{28}$

23 Ebd., Nr. 2135.

24 L. Arbusow (sen.), Livlands Geistlichkeit vom Ende des 12. bis ins 16. Jahrhundert, III. Nachtrag, in: Jahrbuch für Genealogie, Heraldik und Sphragistik 1911/1912 [1913], S. 1-432 (Boddekker, Cristoffer).

25 C. Botticher, Wunderbarliche Handlung deß jetzigen Meysters auß Leyflandt unnd seines Stallbruders oder Coadiutorn Wilhelmen Fürstenberg und irem anhang, Königsberg 1556.

26 Herzog Albrecht von Preußen und Livland (wie Anm. 6), Bd. (1551-1557), Nr. 1910/5.

27 Christopher Bötticher nennt in einem Schreiben aus Vilna 11. Feb. 1556 Fürstenberg schon als Koadjutor, Bergengrün (wie Anm. 5), Beilage 2, S. 307-308.

28 Der Revaler Rat schickt seine Glückwünsche am 1. April 1556 (Regesten aus zwei Missivbüchern des XVI. Jahrhunderts im Revaler Stadt-Archiv, hrsg. v. G. v. Hansen, Reval 1895, Nr. II-220, mit falschem Datum, vgl. TLA, f. 230, n. 1, s. Aa 21, Fol. 251r). Fürstenberg selbst bittet den Deutschmeister um seine Bestätigung am 28. April 1556 (Nordosteuropa und der Deutsche Orden. Kurzregesten, hrsg. v. K. Wieser, Bd. 1: bis 1561, Bad Godesberg 1969, Nr. 1789, 1790). 
Jasper von Munster hat diese Wahl als rechtswidrig bezeichnet. Sein erstes Argument war, dass der Landmarschall als Stellvertreter des Meisters auch Anspruch auf die Nachfolge habe. Wenn man auch in Hinsicht auf den allgemeinen Brauch Jasper Recht geben kann, ist doch zu betonen, dass die Praxis im Orden schon immer vielfältiger war. Unter Jaspers Zeitgenossen ist zum Beispiel Johann von der Recke aus dem Amt des Komturs von Fellin zum Koadjutor gewählt und danach zum Meister erhoben worden. Der andere Vorwurf Jaspers, dass nicht alle Gebietiger an der Wahl teilgenommen haben, ist hingegen ernster zu nehmen. Laut Jasper ist Wilhelm von Fürstenberg von den Komturen zu Reval, Goldingen und Marienburg sowie von den Vögten zu Jerwen, Soneburg, und Kandau gewählt worden. ${ }^{29}$ Diese Liste enthält in der Tat nicht alle Gebietiger, wenn man aber auf die Ämter sieht, sind hier immerhin die gewichtigsten Gebietiger des Livländischen Ordens vertreten. Wen man die direkt Beteiligten, das heißt den Meister, den Landmarschall und den Komtur von Fellin, nicht zählt, bleibt kein einziger der sogenannten Gebietiger des inneren Rates übrig ${ }^{30}$, der an der Wahl noch hätte teilnehmen können. Quellenkritisch ist auch wichtig zu betonen, dass diese Aufzählung der Wahlmänner Fürstenbergs von Jasper selbst stammt, was bedeutet, dass hier eher Namen fehlen, als dass es zu viele sind, bzw. dass der Kreis kleiner dargestellt wird, denn es handelt sich um einen Teil der Argumentation.

Hauptpunkt ist jedenfalls, dass alle andere Gebietiger die Wahl Wilhelms von Fürstenberg als rechtmäßig anerkannt bzw. nicht bestritten haben. Über Meinungsbildungsprozesse und unterschiedliche Gruppierungen auf dem Ordenskapitel wissen wir leider sehr wenig. Genauso schwierig, wie es oben war die Partei Munsters zu rekonstruieren, fällt es schwer die Partei Fürstenbergs zu erfassen. Man hat unter den Ordensgebietigern wohl heftig diskutiert, und es ist möglich, dass bei dieser Koadjutorwahl neben Jasper von Munster auch Namen anderer Gegenkandidaten Fürstenbergs genannt wurden. Jedenfalls behauptet der Chronist Salomon Henning, dass schon damals die Kandidatur seines Herrn Gotthard Kettler vorgeschlagen wurde. ${ }^{31}$ Die endgültige Wahl von Fürstenbergs wird aber nicht in Frage gestellt. Es fehlen auch Beweise dafür, dass irgendein Ge-

29 Herzog Albrecht von Preußen und Livland (wie Anm. 6), Bd. (1551-1557), Nr. 1813 (Franz von Sieghoven gen. Anstel (Reval), Christoph von Neuhof gen. Ley (Goldingen), Philipp Schall von Bell (Marienburg), Bernd von Schmerten (Jerwen), Heinrich Wulff (Soneburg), Heinrich Steding (Kandau)).

30 Zum Begriff: K. Neitmann, Rat und Ratsgebietiger Wolters von Plettenberg. Beobachtungen zum Regierungs- und Verwaltungsstil des Ordensmeisters, in: Wolter von Plettenberg und das mittelalterliche Livland, hrsg. v. N. Angermann, I. Misans, Lüneburg 2001, S. 85-111.

31 S.Henning, Lifflendische Churlendische Chronica, in: Scriptores Rerum Livonicarum, Bd. 2, Riga- Leipzig 1853, S. 218. 
bietiger irgendwie seine Sympathie gegenüber Munster geäußert hätte. Jaspers Versuche, die Koadjutorwahl als unrechtmäßig darzustellen, was Salomon Henning als polypragmosyne bezeichnet ${ }^{32}$, sind im Großen und Ganzen gescheitert. Jaspers Netzwerk unter den Gebietigern war nicht besonders stark, er konnte nur die ihm direkt unterstellten Ordensbrüder wie Johann von der Hoete an sich binden, aber keine tiefere Spaltung des Ordens hervorrufen. Jaspers Streit ist demnach kein ordensinterner Parteienstreit, sondern ein Konflikt zwischen ihm und dem Orden. ${ }^{33}$ Jasper wurde wegen seines Ungehorsams abgesetzt, was er Anfang April als eine vollzogene Tatsache darstellt. ${ }^{34}$

\section{Die Vertreibung von Jasper}

Obwohl die politischen Netzwerke Jaspers zerrissen waren, blieb er bis Anfang Mai in Livland. Es scheint, dass Jasper versuchte, sich zunächst in seinem Gebiet zu verschanzen; er hoffte jedenfalls auf einen Angriff von der Seite Polen-Litauens. Das Gebiet des Landmarschalls lag in Zentrallivland in drei getrennt voneinander liegenden Teilen um Segewold, Mitau und Ascheraden (siehe die Karte S. 82). Nach Jaspers späterer Einschätzung waren ihm 7 Burgen und 8 Höfe mit insgesamt 1200 Personen unterstellt. ${ }^{35}$ Der Landmarschall konnte insgesamt ungefähr 300 Reiter ins Feld bringen ${ }^{36}$, eine beträchtliche Machtbasis jedenfalls. Ende April tauchte im Gebiet Goldingen der ehemalige Landschreiber aus Segewold auf und berichtete, dass der Feind bald angreife. Wer der Feind war und woher er kommen sollte, war dem Komtur von Goldingen damals noch nicht klar, doch forderte er alle Untertanen des Ordens zur Kriegsbereitschaft auf. ${ }^{37}$ Das Milieu war ohnehin nervös. Der Komtur von Dünaburg, Gotthard Kettler, war in Deutschland um

32 Ebd.

33 Lange (wie Anm. 2), S. 238, bezeichnet Munsters verhalten als Alleingang, was zuzustimmen ist.

34 Herzog Albrecht von Preußen und Livland (wie Anm. 6), Bd. (1551-1557), Nr. 1813.

35 Ebd., Bd. (1557-1560), Nr. 2504.

36 So Henning (wie Anm. 31), S. 269. Siehe auch F. Benninghoven, Probleme der Zahl und Standortverteilung der livländischen Streitkräfte im Ausgehenden Mittelalter, Zeitschrift für Ostforschung 12 (1963), H. 3, S. 601-622, hier S. 605. Die von Benninghoven im Anhang S. 620-622 publizierte Liste, vgl. auch Herzog Albrecht von Preußen und Livland (wie Anm. 6), Bd. (1551-1557), Nr. 1711, ist mit Sommer $1556 \mathrm{zu}$ datieren, weil darin der Landmarschall als nicht anwesend und vilele Ordensgebietiger als bei Kokenhusen liegend bezeichnet sind.

37 SRA Livonica I: 17 (Komtur von Goldingen Christoffer von Neuhof an Ordensmeister, 29. April 1556, Zabeln). 
Knechte anzuwerben, und während des Landtages in Wolmar war im Himmel ein Komet zu sehen. ${ }^{38}$

Zuerst schien es so, als ob der Orden den abgesetzten Landmarschall nur durch den offenen Krieg loswerden könnte. In der Spätzeit des Deutschen Ordens in Livland wurde der mangelnde Zugriff der Zentrale auf die Ordensgebiete und die wachsende Selbstständigkeit der Gebietiger gewiss zum Problem. Die Koadjutorfehde ermöglicht es die Grenzen dieser Selbstständigkeit zu erforschen und zu fragen, wie sich das administrative Netzwerk des Ordens während der Fehde verhalten hat.

Als Jasper seine Hauptburg Segewold verließ, um in Dünamünde Station zu machen, wurde ihm der Zugang auf Befehl des Meisters vom dortigen Hauskomtur Georg von Brabeck ${ }^{39}$ verweigert. Der Abzug Jaspers aus Segewold wurde später von Johann von Hoete fast als Verschwörung dargestellt. Es war laut Hoete der Kumpan des Landmarschalls, Wiegant von Scharenberg ${ }^{40}$, der empfahl sich aus Segewold nach Dünamünde zu begeben, obwohl er schon wusste, dass Jasper nicht eingelassen werden würde. ${ }^{41} \mathrm{~W}$ iegant hat später die Beweise geliefert, wie Jasper und Johann von Hoete den auswärtigen Angriff gegen Orden geplant haben. ${ }^{42}$ Wiegant als Hauptkraft der Verschwörung gegen Jasper darzustellen, wäre aber doch zu viel des Guten; Wiegant ist später nur als einfacher Ritterbruder in Trikaten belegt. Der Hauskomtur von Dünamünde, Georg von Brabeck, wurde aber für seine Standhaftigkeit belohnt und ins das neugeschaffene Amt des Komturs von Segewold befördert, wobei gleichzeitig der frühere Hauskomtur zu Segewold im Amt blieb. ${ }^{43}$

Nach dem Misserfolg in Dünamünde zog Munster näher an die litauische Grenze, wo seine Burg Ascheraden lag. Laut den Angaben des dortigen Hauskomturs Wilhelm Holtey ${ }^{44}$ kam er am Abend des 3. Mai mit einem Gefolge von 74 Reitern bei der Burg an. Eine Delegation, geleitet von Johann von Hoete, ging zum Hauskomtur, um im Namen des Landmarschalls Eintritt zu fordern. Zur Delegation gehörten der Domherr von Riga und Jaspers Vetter Johann von Munster sowie die Vertreter der örtlichen Ordensvasallen, Wolmar Üxkull, Georg Stich-

38 Diese sogenannte Komet Karls V. ist auch in mehreren livländischen Chroniken erwähnt. Vgl. J. Renner, Livländischen Historien 1556-1561, hrsg. v. P. Karstedt, Lübeck 1953, S. 8; B. Russow, Chronica der Poruintz Lyfflandt, in: Scriptores Rerum Livonicarum, Bd. 2, S. 48.

39 Ritterbrüder (wie Anm. 7), Nr. 102.

40 Ebd., Nr. 766.

41 Herzog Albrecht von Preußen und Livland (wie Anm. 6), Bd. (1557-1560), Nr. 2137.

42 Ebd.

43 Ritterbrüder (wie Anm. 7), Nr. 963 (Bernhard von Wevorden).

44 Ebd., Nr. 436. 


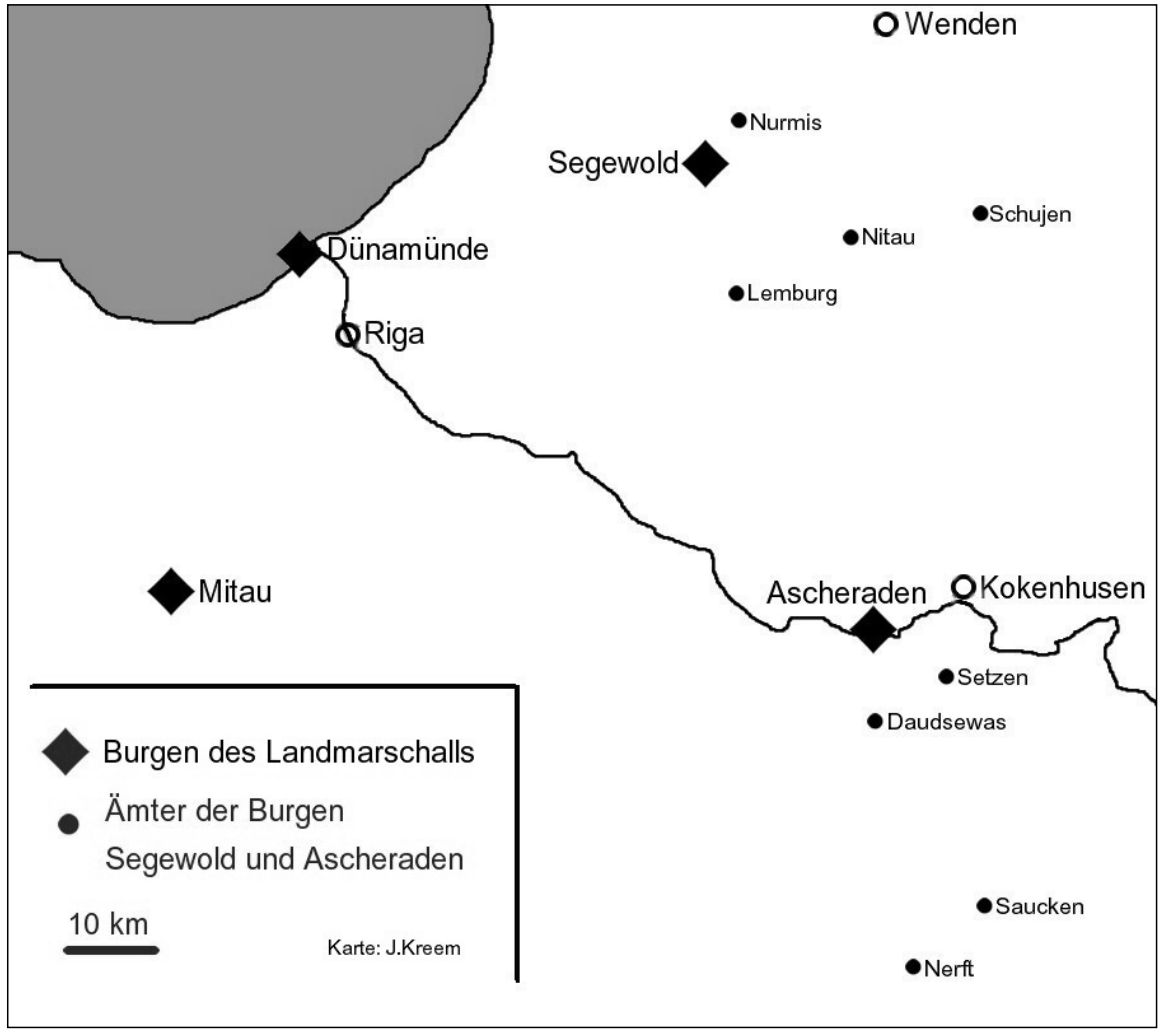

horst und Klaus Hastfer. Auch der Hauskomtur von Ascheraden verweigerte dem Landmarschall den Einzug in die Burg. ${ }^{45}$ Am 4. Mai zog Jasper weiter. ${ }^{46}$ Holtey bekam für seine Treue gegenüber dem Orden keine unmittelbare Belohnung. Im Jahre 1558 wollte ihn Fürstenberg zum Vogt von Grobin ernennen, aber er konnte nicht mehr ins Amt eintreten. ${ }^{47}$

Während Jasper selbst mit einem kleinen Gefolge Erzbischof Wilhelm in Kokenhusen aufsuchte, ritt der größte Teil seiner Truppen über die Düna nach Setzen. ${ }^{48}$ Diesen Gutshof, einen der Amtsbezirke der Burg Ascheraden, hat Jasper gründlich geplündert und den dortigen Landknecht (bzw. den Verwalter) zusam-

45 SRA Livonica I: 17 (Hauskomtur von Ascheraden an Ordensmeister, 4. Mai 1556).

46 SRA Livonica I: 17 (Hauskomtur von Ascheraden an Ordensmeister, 5. Mai 1556).

47 Ritterbrüder (wie Anm. 7), Nr. 436.

48 Herzog Albrecht von Preußen und Livland (wie Anm. 6), Bd. (1551-1557), Nr. 1822, 1824. Vgl. Renner (wie Anm. 38), S. 10-11. 
men mit dessen Dienerschaft zum Treueid gezwungen. Es soll insgesamt eine etwa 90 Reiter starke Einheit entstanden sein. ${ }^{49}$ Erzbischof Wilhelm berichtete, dass eine Ordenseinheit von 400 Reitern Munster nachgejagt sei, demgegenüber klagte der Hauskomtur von Ascheraden, er sei zu schwach, um Jasper aufzuhalten. Es ist jedenfalls gerade dieser Zeitpunkt, den wir als militärische Eskalation bezeichnen können. Jasper schätzte seine Position in Setzen als zu schwach ein und zog irgendwann nach dem 10. Mai in Richtung Litauen ab. ${ }^{50}$ Am 23. Mai war er schon in Kaunas. ${ }^{51}$

Direkt nach der Flucht von Jasper haben die Hauskomture von Segewold und Ascheraden eine Bilanz an den Ordensmeister geschickt. Aus dem Burggebiet Segewold war der Landsknecht von Lemburg mit Jasper mitgegangen..$^{52} \mathrm{Im}$ Gebiet Ascheraden hatte der Landsknecht von Setzen dem Landmarschall Treue geschworen. ${ }^{53}$ Die anderen Landsknechte wurden von den Hauskomturen zum Gehorsam gegenüber dem Orden überredet. Die Verbindungen innerhalb des Beamtennetzwerks werden in den Briefwechsel der Zeit mit dem Konzept der Eidespflicht beschrieben. Während die Ordensüberlieferung vom Eid gegenüber dem Orden spricht, sieht Erzbischof Wilhelm die Diener durch ihren Eid an die Person Jaspers gebunden. ${ }^{54}$ Diese unterschiedlichen Auffassungen trafen in der Koadjutorfehde aufeinander, wobei wir die Stärke und Reichweite des administrativen Netzwerks des Ordens feststellen können. Die Kette vom Ordenskapitel am Landmarschall vorbei über die Hauskomture ihres Gebiets bis hin zu den einzelnen Amtmännern in den Gutshöfen konnte von Jasper von Munster nicht gebrochen werden. Jasper konnte in seiner unmittelbaren Umgebung immerhin noch eine beträchtliche Anzahl von Männern, Vasallen und Dienern als eine Art von Hof oder Klientel an sich binden. Für die Beherrschung des gesamten Gebiets reichte das aber nicht aus. Als er seine Residenz Segewold verließ, konnte er keine Burg mehr als Stützpunkt gewinnen.

49 SRA Livonica I: 17 (Hauskomtur von Ascheraden an Ordensmeister, 12. Mai 1556).

50 Am 12. Mai schreibt Wilhelm, dass Munster in der nächsten Nacht weiterzieht: Herzog Albrecht von Preußen und Livland (wie Anm. 6), Bd. (1551-1557), Nr. 1827.

51 Ebd., Nr. 1833. In der Verteidigungsschrift von Jasper von Munster (wie Anm. 15) ist die Chronologie verzerrt wiedergegeben.

52 SRA, Livonica I: 17 (Hauskomtur von Segewold an Ordensmeister, 8. Mai 1556).

53 SRA, Livonica I: 17 (Hauskomtur von Ascheraden an Ordensmeister, 12. Mai 1556).

54 SRA, Livonica I: 17 (Hauskomtur von Ascheraden an Ordensmeister, 12. Mai 1556); Herzog Albrecht von Preußen und Livland (wie Anm. 6), Bd. (1551-1557), Nr. 1822; Renner (wie Anm. 38), S. 10. 


\section{ESKALATION UND EPILOG}

Die weitere Eskalation können wir hier nur kursorisch verfolgen. Die Klageschrift des Ordens vom Ende Mai trägt das Motto: retribuat Dominus facienti malum iuxta malitiam suam (2 Samuel, 3, 39: Der Herr vergelte dem, der Böses tut, nach seiner Bosheit). ${ }^{55}$ Der Orden stellte den Landmarschall und Erzbischof Wilhelm negativ dar. Sie hätten diese lande nicht allein in ibren gewalt zubringen vorhabens, sondern auch dieselben von aller ihrer alten loblichen lieberteten, herligkeit und freiheit zubringen, und mit allerlei servituten, dienstbarkeiten und anderen unzimlichen burden zubeschweren vnd zubeladen gedencken, wie dan vns Preussen und andere ein exempell und spiegell sein magk. ${ }^{56}$ Die livländischen Stände waren aufgrund der Flucht des Landmarschalls über Kokenhusen nach Litauen schnell davon überzeugt, dass der Landmarschall mit dem Erzbischof in eine Konspiration mit Polen-Litauen verwickelt war. Zusätzliche Beweise lieferte die entwendete Korrespondenz zwischen Wilhelm und Herzog Albrecht. ${ }^{57}$ Für den Ausbruch der Koadjutorfede des Rigaer Erzbistums war Jasper gewiss nicht der einzige Schuldige, seine Flucht hat aber ohne Zweifel zu deren Eskalation merklich beigetragen. ${ }^{58}$

Der Orden hatte sich schon gegen den Landmarschall gerüstet, es lag auf der Hand, dieselben Kräfte gegen den Erzbischof zu benutzen, zumal in Livland ein breiter Konsens gegen Wilhelm herrschte. Ende Juni wurde Kokenhusen erobert, und der Krieg innerhalb Livlands war zu Ende. Die Absetzung Wilhelms war für Sigismund August von Polen als Protektor des Erzstiftes ein legitimer Anlass zum Eingreifen. Das Problem des Landmarschalls aber war und blieb nach Einschätzung der Räte des Königs eine interne Sache des Ordens ${ }^{59}$; es wurde daher nicht in den Friedensvertrag von Poswol zwischen Livland und Polen-Litauen aufgenommen. Bei den späteren Restitutionsverhandlungen wurde viel geredet und versprochen, doch blieb die Restitution des Landmarschalls aus. Nach dem Ausbruch des Livländischen Krieges 1558 wurden die Möglichkeiten des Ordens irgendetwas zur Versorgung Jaspers zu finden mit jedem Kriegsmonat geringer. Erst im Jahre 1562 wurde die Vergabe der Burg Ascheraden an Jasper verfügt, damals war der Orden aber schon aufgelöst. ${ }^{60}$

\footnotetext{
55 SRA, Livonica I: 42 (28. Mai 1556, Wenden).

56 Ebd.

57 Renner (wie Anm. 38), S. 10.

58 Lange (wie Anm 2), S. 205.

59 Herzog Albrecht von Preußen und Livland (wie Anm. 6), Bd. (1551-1557), Nr. 1816.

60 Ebd., Bd. (1560-1564), Nr. 3014.
} 


\section{ZUSAMMENFASSUNG}

Der ordensinterne Streit in der ersten Jahreshälfte 1556 ermöglicht uns ein Einblick ins Funktionieren der politischen und administrativen Netzwerke während der Spätzeit des Deutschen Ordens. Der Orden agierte während dieses Streits als Korporation. Auch wenn es auf dem Kapitel Meinungsverschiedenheiten gab, hielten sich die Gebietiger an dessen Beschlüsse. Die Beziehungen Jaspers von Munsters konnten innerhalb des Ordens kein Netzwerk schaffen, das das Kapitel gespalten hätte. Die Weigerung Jaspers die Koadjutorwahl anzuerkennen und sein Versuch die Vorgänge als rechtswidrig darzustellen führten zu seinem Bruch mit dem Orden, ohne dass ihm ein Gebietiger darin gefolgt wäre. Der Sturz des Landmarschalls hat wenig mit seiner außenpolitischen Orientierung (polenfreundliche Stimmungen gab es im Orden auch später), sondern vor allem mit seinem Alleingang zu tun.

Bei der faktischen Entfernung des Landmarschalls aus dem Amt sehen wir das administrative Netzwerk des Ordens in Tätigkeit. In dem direkt dem Landmarschall unterstellten Gebiet haben die Hauskomture der Burgen Dünamünde und Ascheraden der Ordensleitung gehorcht und Jasper widerstanden. Jaspers Fähigkeit um sich ein Gefolge von fast Hundert Männern zu sammeln ist jedenfalls beeindruckend und zeigt die Anziehungskraft eines Gebietigers. Die aus Dienern und einigen Vasallen geformte Klientel Jaspers von Munster hat ihm Folge geleistet, und auf dem flachen Lande hat Jasper dazu noch einige Amtmänner unter diesen unsicheren Umständen zum Mitreiten gezwungen, wobei Jaspers persönliche Präsenz ausschlaggebend gewesen zu sein scheint. Für eine längere Behauptung seines Gebiets hat es aber nicht gereicht. Für die Mehrheit seiner Beamtenschaft zählte die Legitimität der Gesamtorganisation damals noch mehr.

\section{SUMmARY \\ Networks around Jasper von Munster. Teutonic Order during the Livonian War of Coadjutors in 1556}

Livonian war of coadjutor's broke out when the estates protested against the election of Christopher of Mecklenburg as a coadjutor of archbishop of Riga. At the same time, another conflict occurred, when Land Marshal of the Teutonic Order in Livonia, Jasper von Munster protested against the election of Wilhelm von Fürstenberg as the coadjutor of the Livonian Master. As a result of the latter, Jasper was deposed and had to flee to Kaunas, from where he fought in vain for his restitution. 
This article aims first to identify and analyse the political network supporting Jasper inside of the Teutonic Order, and second to study the way the administrative network of the Order functioned during the conflict. It appears that Jasper's political connections did not suffice to form his "party", which could divide the chapter of the Order. During the conflict, the officials of the Order mostly retained their loyalty to the organization. Jasper was able to form a small retinue or a court of a kind, which consisted of local nobility, minor officials and even some fellow brethren of the Order, but it was not enough to confront the rest of the Order. 